\title{
Measurement Based Validation of an Electro-Pneumatic Gearbox Actuator
}

\author{
Ádám Szabó1*, Tamás Bécsi ${ }^{1}$, Szilárd Aradi \\ 1 Department of Control for Transportation and Vehicle Systems, Faculty of Transportation Engineering and Vehicle Engineering, \\ Budapest University of Technology and Economics, H-1111 Budapest, Stoczek u. 2, Hungary \\ * Corresponding author, e-mail: szabo.adam@mail.bme.hu
}

Received: 25 April 2019, Accepted: 13 May 2019, Published online: 31 October 2019

\begin{abstract}
The objective of the research is to analyze the behavior of the developed electro-pneumatic actuator model and compare it to the behavior of the real system. The actuator achieves the requested gear changes by moving the two pistons inside the cylinder and it is operated by three-way two-position solenoid valves. Since not all model parameters are exactly known, such as contraction coefficients and friction parameters, they can be estimated based on literature then they can be further tuned to minimize the error of the simulation. The developed nonlinear model is capable of describing the dynamic behavior of the gearbox actuator, thus it can be used to analyze the effects of constructional modifications and it can serve as Model in the Loop (MIL) environment for controller testing. Keywords
\end{abstract}

pneumatic actuators, nonlinear systems, validation

\section{Introduction}

Model-based development (MDP) is a widely used method in designing control systems and an effective way to react to the increasing product complexity and decreasing development time.

MDP has a wide range of applications, such as modeling of power networks for voltage controls (Petrone et al., 2012), development of communication systems (Duncan and Etienne-Cummings, 2017), modeling of fuel cell systems (Ishaku et al., 2014) and it is of great importance for the automotive industry as it can be seen in (Bringmann and Krämer, 2008; Ogata and Katayama, 2010; Wakitani and Yamamoto, 2017).

It supports the development process (V-model) with the application of Model in the Loop (MIL) and Hardware in the Loop (HIL) environments. MIL environments can be used to determine the system specifications based on the given requirements, such as in (Kako et al., 2007) and controller algorithms can also be designed and tested through MILs as it can be seen in (Németh and Gáspár, 2010; Ohata, 2009; SantillanGalvan et al., 2004), then they can be tested in HILs (Deng et al., 2008; Mihály et al., 2017; Rabinovici et al., 2012).

Electro-pneumatic systems are widely used in heavy-duty vehicles since they have many advantages, but their nonlinear behavior makes predicting and controlling them difficult.
Models can be powerful tools in controller and system design, but they should always be developed with regard to their applications and the modeling aims. Finite element models can simulate systems with high accuracy, but they cannot be used to analyze the effects of smaller constructional changes because of their high calculation cost, and they cannot serve as MIL environments. Models without exact physical equations can be used for simple tests, although they are inadequate for tests that are more detailed and for fine tuning the controllers.

Models developed to serve as MIL environments should be able to describe the nonlinear behavior of the system, yet they should have reduced complexity. To provide reliable feedbacks to both design and controller development they have to be verified and validated against laboratory measurements.

The aim of this paper is to show the detailed verification and validation process of the nonlinear model of a pneumatic actuator developed in (Szabó et al., 2018).

The paper is organized as follows: Section 2 describes the modeled actuator, Section 3 shows the model verification, Section 4 presents the validation results and Section 5 shows some conclusion remarks. 


\section{System description}

The function of the modeled actuator is to shift the requested gear within an automated manual transmission of a heavy-duty vehicle, or to set the gearbox to neutral.

The actuator is driven by two 3-way 2-position solenoid valves, which connect the chambers to the supply pressure or to the ambient pressure. There are two pistons, two working chambers and one control chamber within the cylinder, which serves as an air spring between the pistons. The simplified layout of the modeled system can be seen in Fig. 1.

The main piston has three dedicated positions: two gears (High and Low end positions) and a Neutral position, while the floating piston can move only between Neutral and High positions.

The shift fingers main function is force transmission as it connects the actuator to the gearshift linkage. The detent mechanism - which prevents the unintended movement of the pistons - is also connected to the shift finger.

\section{Verification}

The model verification is performed by comparing the behavior of the real system with the simulation results as regards the trends-, operation domain- and relationship of the variables. In the verification process four different operation cases were considered, which are the gear-to-neutral and neutral-to-gear changes. The verified outputs are the working chamber pressures and the main piston position.

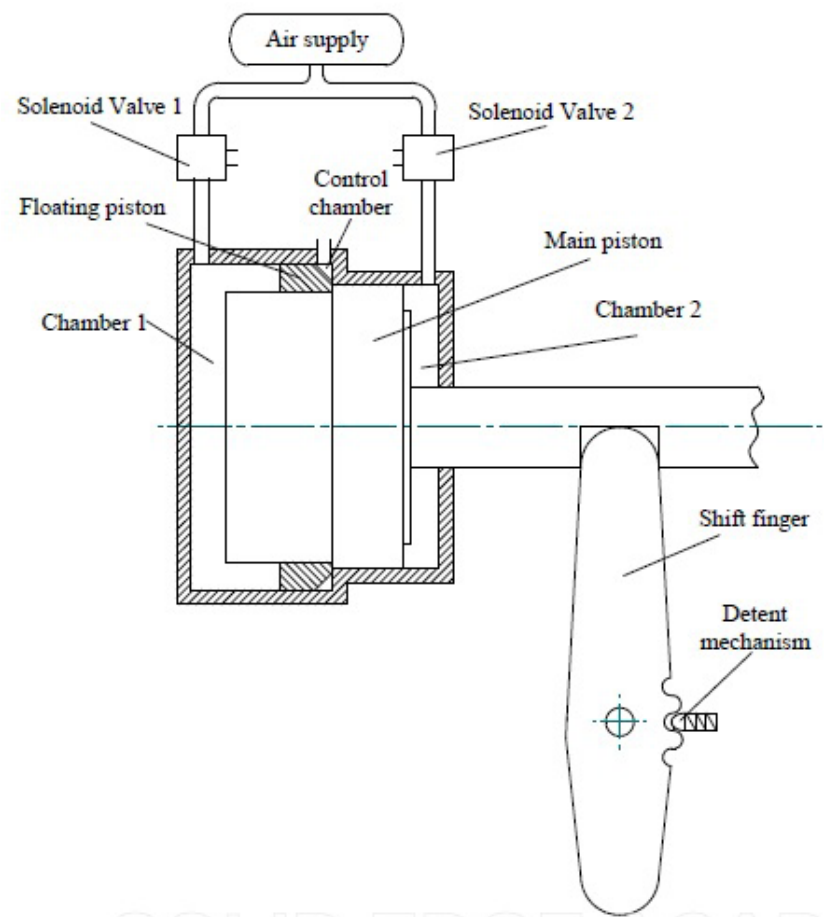

Fig. 1 Simplified layout of the gearbox actuator

\subsection{Neutral to High gear change}

The first test case can be seen in Fig. 2. To switch the actuator from Neutral to High position Solenoid Valve 1 is energized. It can be seen that after approximately $3 \mathrm{~ms}$ delay - which is caused by the inertia of the solenoid valve - the pressure in Chamber 1 starts to increase and after reaches, the supply pressure it remains constant. After releasing the solenoid valve the air from Chamber 1 exhausts, thus its pressure sets back to the ambient pressure.

When the pressure difference between the chambers is high enough to overcome the Coulomb-friction both the Main- and Floating pistons start to move towards High position. After reaching their end position, the movement of the pistons stops and a breakpoint can be observed in Chamber 1 pressure since the volume change of Chamber 1 suddenly drops to zero.

A small pressure increase can also be seen in Chamber 2, which is caused by the combined effect of its decreasing volume and the choke of Solenoid valve 2 .

\subsection{High to Neutral gear change}

A High to Neutral gear change can be seen in Fig. 3. To reach Neutral position both solenoid valves were energized at the same time. Chamber 2 has smaller volume at the beginning of gear change, therefore the pressure gradient of this chamber is higher, than the pressure gradient
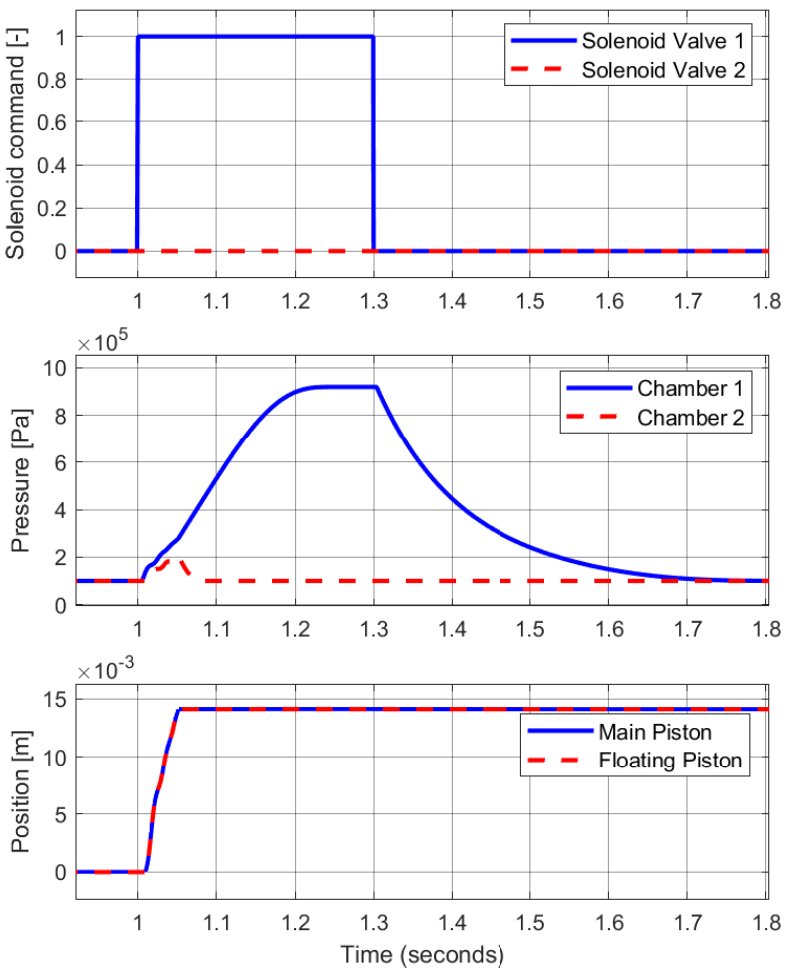

Fig. 2 Verification - Neutral to High gear change 

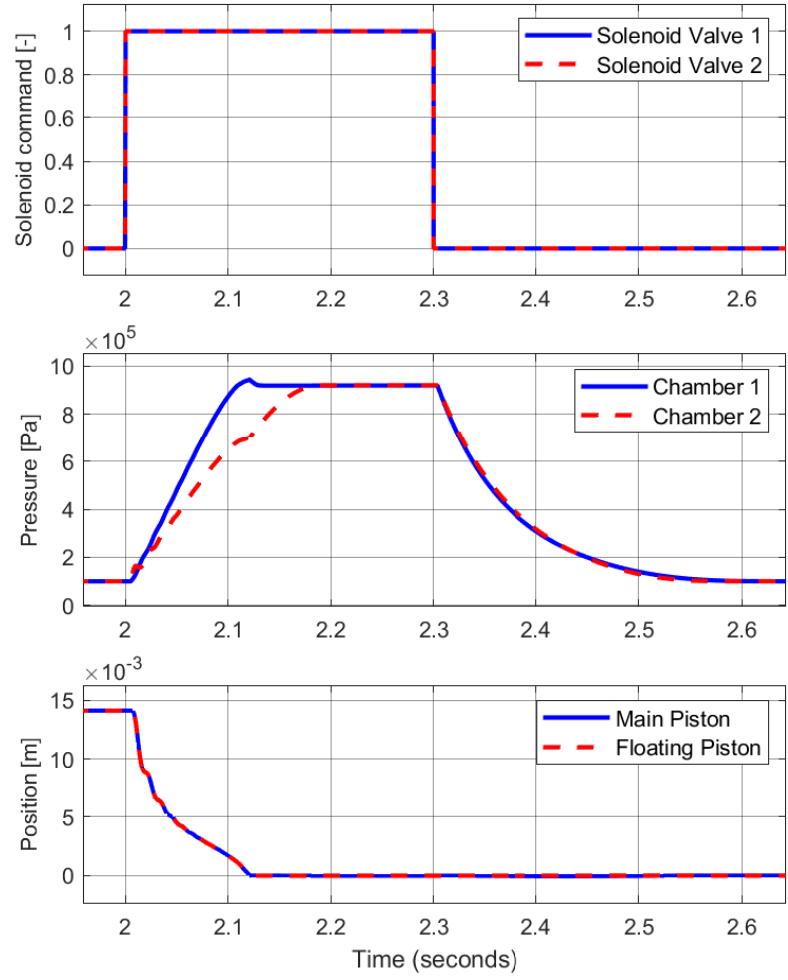

Fig. 3 Verification - High to Neutral gear change

of Chamber 1. However, as the pistons start to move, the volume decrease of Chamber 1 compensates the smaller initial volume of Chamber 2, thus Chamber 1 pressure is higher for the most part of the gear change.

It can be seen that for a short time the pressure of Chamber 1 exceeds the supply pressure, which is caused by the decreasing volume of the chamber as its pressure reaches the supply pressure before the movement stops.

Since Chamber 2 pressure is applied not only to the Main Piston, but also to the Floating Piston the smaller pressure inside Chamber 2 can switch the actuator to Neutral.

\subsection{Neutral to Low gear change}

The third test case can be seen in Fig. 4. In this case, most of the conclusions that were made regarding the first test case are also correct. The only relevant difference between the two cases can be seen in the behavior of the Floating Piston. As the movement of the Floating Piston is limited between Neutral and High, in this case it stays in Neutral position as it was expected.

\subsection{Low to Neutral gear change}

The last test case can be seen in Fig. 5. It shows that the movement of the Main Piston is slower, than in case of the second test case because in this side of the actuator there is no floating piston. Therefore, the pressure force
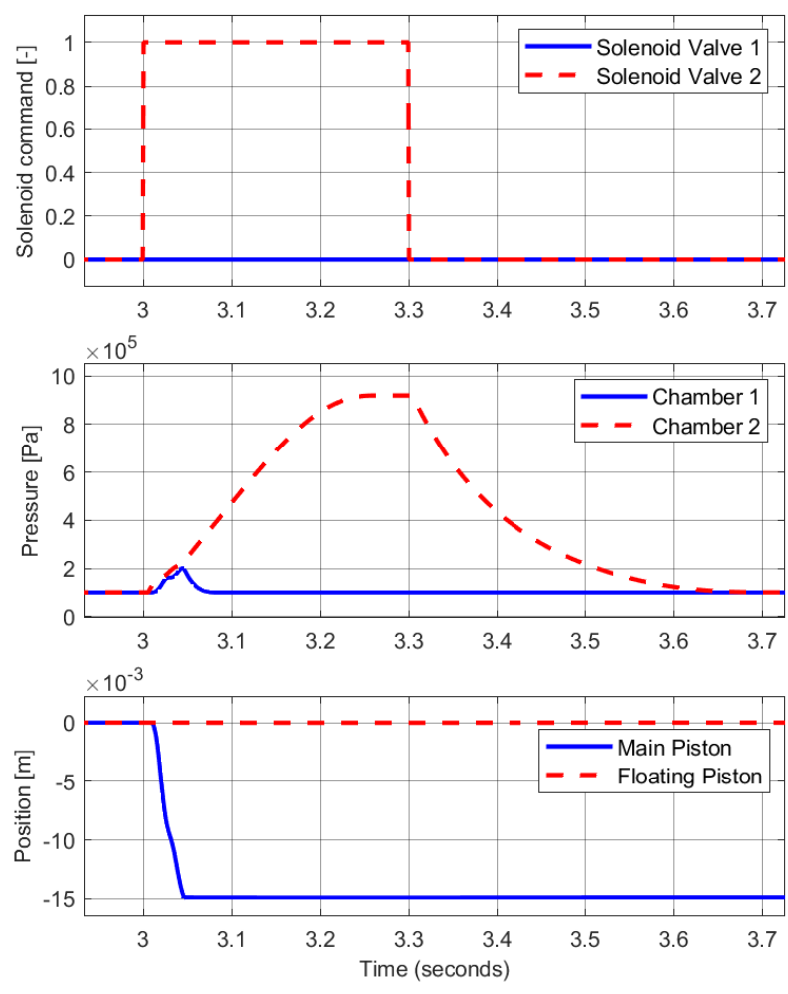

Fig. 4 Verification - Neutral to Low gear change
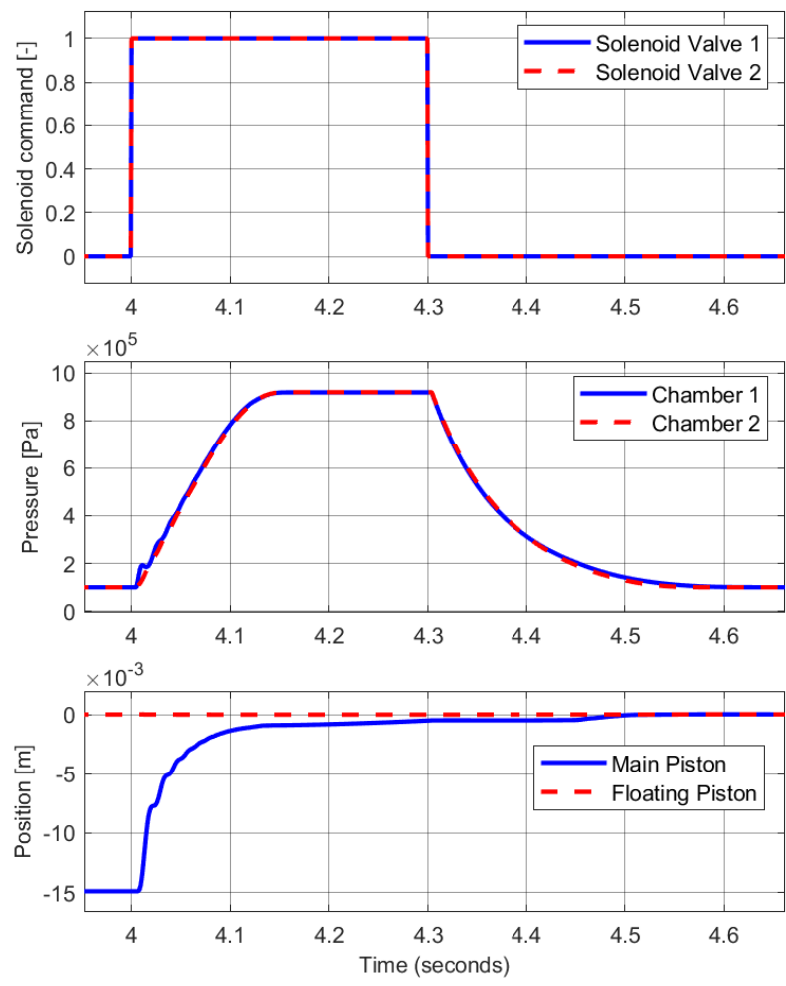

Fig. 5 Verification - Low to Neutral gear change

generated by the pressure difference of the working chambers is much lower as the differences of the corresponding piston areas are smaller. This can be compensated by an offset between the solenoid commands. 


\section{Validation}

\subsection{Measurement system}

The model was validated against laboratory measurements, where the measured signals were the solenoid commands, the working chamber pressures and main piston position.

The measurements were taken on a heavy-duty AMT test bench, which includes a 16-speed heavy-duty automated gearbox, a gearbox actuator (including the shift cylinder), two electric motors with speed sensors, a supply pressure sensor and for each cylinders in the actuator there are two pressure sensors and a position sensor.

During the validation measurements the supply pressure was set to 9.5 bar and the supply voltage was $28 \mathrm{~V}$. The chamber pressures were measured with $2 \mathrm{~ms}$ sample time, while the piston position was measured with $5 \mathrm{~ms}$ sampling. In the validation process all four possible gear changes were analyzed.

\subsection{Parameter estimation}

The aim of the validation was to minimize the sum squared error of the simulation through tuning the unknown parameters of the model.

First, the unknown parameters of the model had to be determined. These are the contraction coefficients, heat transfer coefficients, viscous friction coefficients and Coulomb friction parameters, then for each of them an initial value was defined along with its extremes. A constant delay was also taken into consideration for each sensor.

In the next step the thermodynamic (including the solenoid valve models) and mechanical models were validated separately. The sample times of the measurements are much higher, than the simulations, thus this step can only serve as a rough parameter estimation before finetuning the model.

In case of the thermodynamic model the input signals were the solenoid commands and the piston positions, where the floating piston position was determined based on the main piston position. Because of the relatively large sample time of the position measurement, the measured signal had to be interpolated. This sub model could have been further tuned by fixing the pistons in their end positions, hence further increasing the accuracy of the parameter estimation. The tuned parameters of the thermodynamic model were the contraction coefficients of the solenoid valve and the heat transfer coefficients of the cylinder.

To validate the mechanical model, the input signals were chosen as the measured working chamber pressures, while the control chamber pressure was assumed equal to the ambient pressure. The identified parameters of the mechanical model were the friction related parameters of the model.

Parameters identified with the standalone validation of the sub models were used as initial values and they were further tuned during the validation of the complete model.

\subsection{Validation results}

The Neutral to Gear and Gear to Neutral gear changes can be seen in Fig. 6, Fig. 7, Fig. 8 and Fig. 9. The figures presented in Subsection 4.3 show the results of the validation and parameter identification with solenoid commands as input of the model and should be interpreted as follows: the first diagram shows the solenoid valve commands, the second and third diagrams present Chamber 1 and Chamber 2 pressures $\left(\mathrm{p}_{\mathrm{ch} 1}\right.$ and $\left.\mathrm{p}_{\mathrm{ch} 2}\right)$ and the fourth diagram shows the main piston position $\left(\mathrm{x}_{\mathrm{mp}}\right)$.

Fig. 10 shows the sum squared error of the model after validation in every measured timestep.

To get a representative value for the accuracy of the model the Euclidean norm of each error signals was calculated as follows:

$\varepsilon_{y, i}^{j}=\sqrt{\frac{1}{T} \int_{0}^{T} \frac{y_{i, \text { meas }}^{j}(t)-y_{i, \text { sim }}^{j}(t)}{y_{i, \text { meas }}^{j}-y_{i, \text { meas }}^{j}} d t .}$

All validated outputs have their own time interval for every test case, which is limited to the time, when the given signal varies, hence the constant states cannot distort the results. Individual errors of the model can be seen in Table 1, where $N$ refers to Neutral, $H$ refers to High and $L$ refers to Low.

\section{Conclusion}

It can be seen in Table 1 that the model accuracy is above $95 \%$ for all three validated outputs for all test cases. The standalone test cases can be grouped and compared in different ways, for instance a useful comparison can be made between Gear to Neutral and Neutral to Gear shifts.

Instead of a more complex friction model, such as the Stribeck model, a combination of Coulomb- and viscous friction was implemented. During Gear to Neutral gear

Table 1 Euclidean norms of the error signals

\begin{tabular}{lcccc}
\hline & $\mathrm{N} 2 \mathrm{H} \mathrm{f}$ & $\mathrm{H} 2 \mathrm{~N} \mathrm{1}$ & $\mathrm{N} 2 \mathrm{~L} \mathrm{1}$ & L2N f \\
\hline $\mathrm{p}_{\mathrm{ch} 1}[\%]$ & 1.45 & 2.96 & 1.84 & 2.28 \\
$\mathrm{p}_{\mathrm{ch} 2}[\%]$ & 1.02 & 4.49 & 1.91 & 4.57 \\
$\mathrm{x}_{\mathrm{mp}}[\%]$ & 3.13 & 3.38 & 2.40 & 2.69 \\
\hline
\end{tabular}



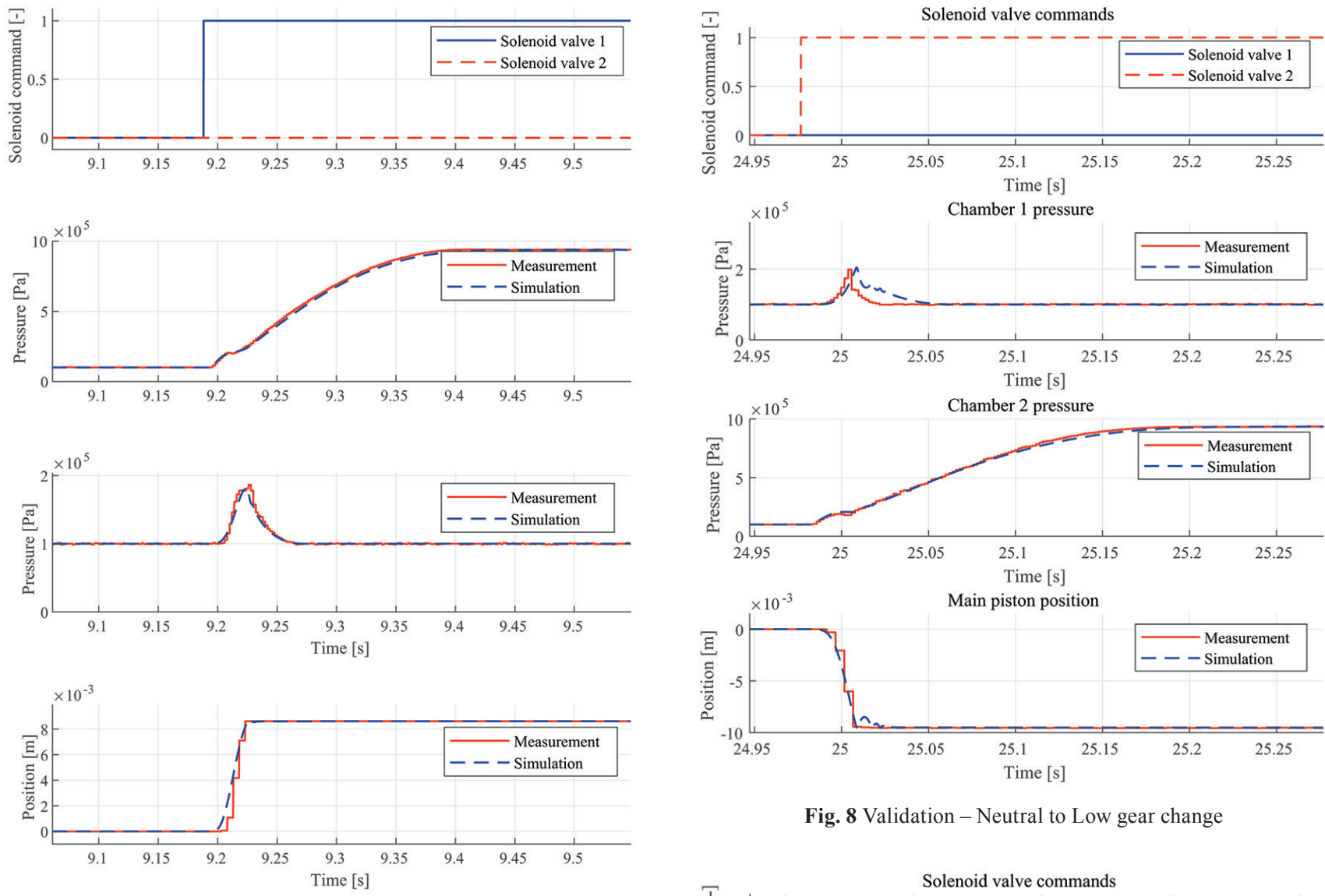

Fig. 8 Validation - Neutral to Low gear change

Fig. 6 Validation - Neutral to High gear change
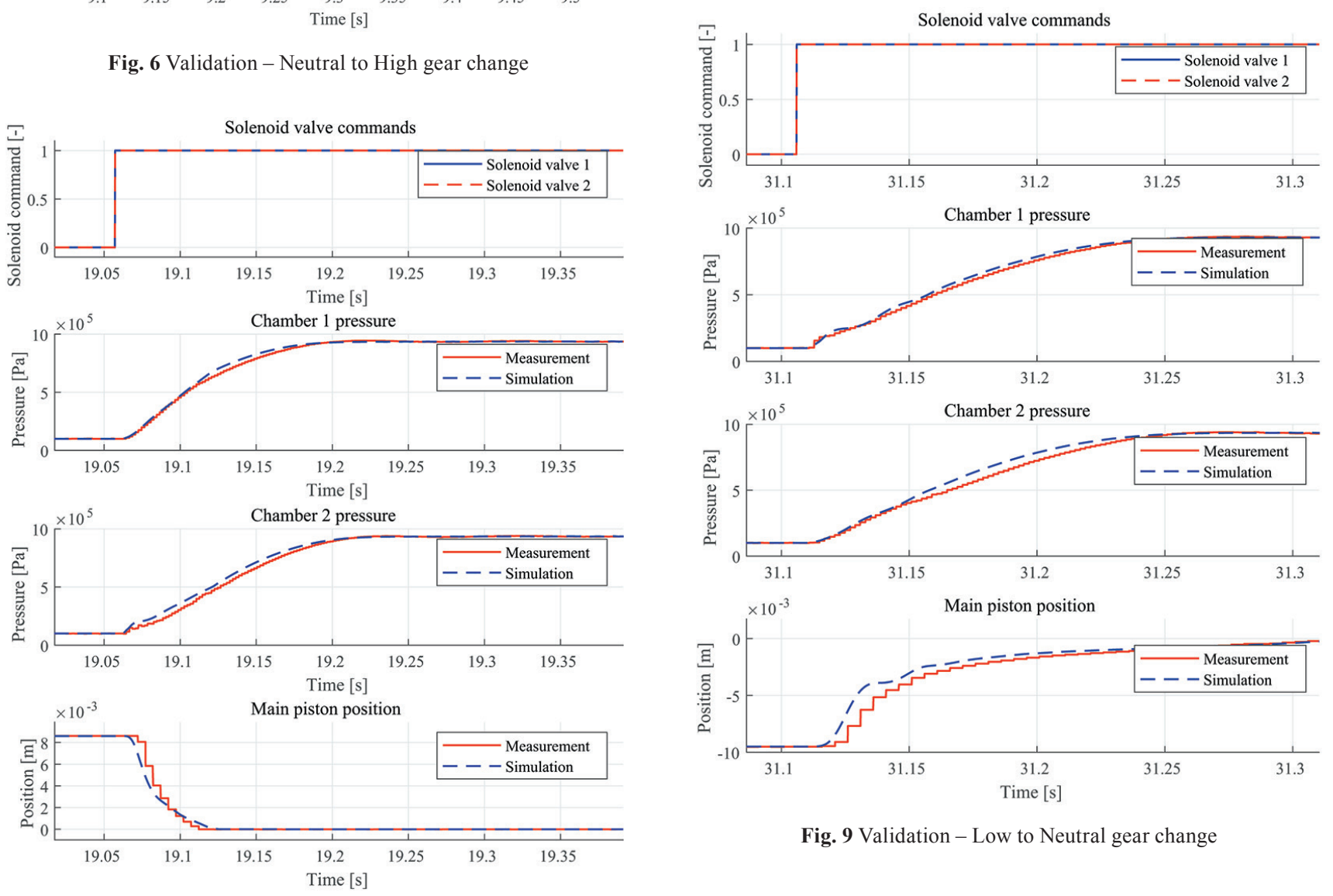

Fig. 9 Validation - Low to Neutral gear change

Fig. 7 Validation - High to Neutral gear change 
changes the effect of the breakaway friction can be much higher as the movement of the pistons is slower, than in case of Neutral to Gear shifts, which can explain why are the latter test cases more accurate. Another possible reason could be that the supply lines of the working chambers were not perfectly separated during the measurement, which can distort the results. This would also explain the small pressure increments in both of the working chambers, which can be observed when air was exhausted from the counter-side chamber and there is no piston movement. This can be seen in Fig. 10 around $12 \mathrm{~s}$ and $27 \mathrm{~s}$.
The results can also be compared based on the location of the main piston. As between Low and Neutral the control chamber has no effect and there is no collision, only friction between the pistons, the higher accuracy of the simulated position meets the prior expectations.

It can be seen, that the pressure deviances are much smaller in case of Neutral to High gear changes, than in any other test cases. This can be explained by the Main Pistons geometry. On the right side of the cylinder the piston movement is limited by a bumper, while on the left side it is limited by the piston skirt. The exhaustion is assured
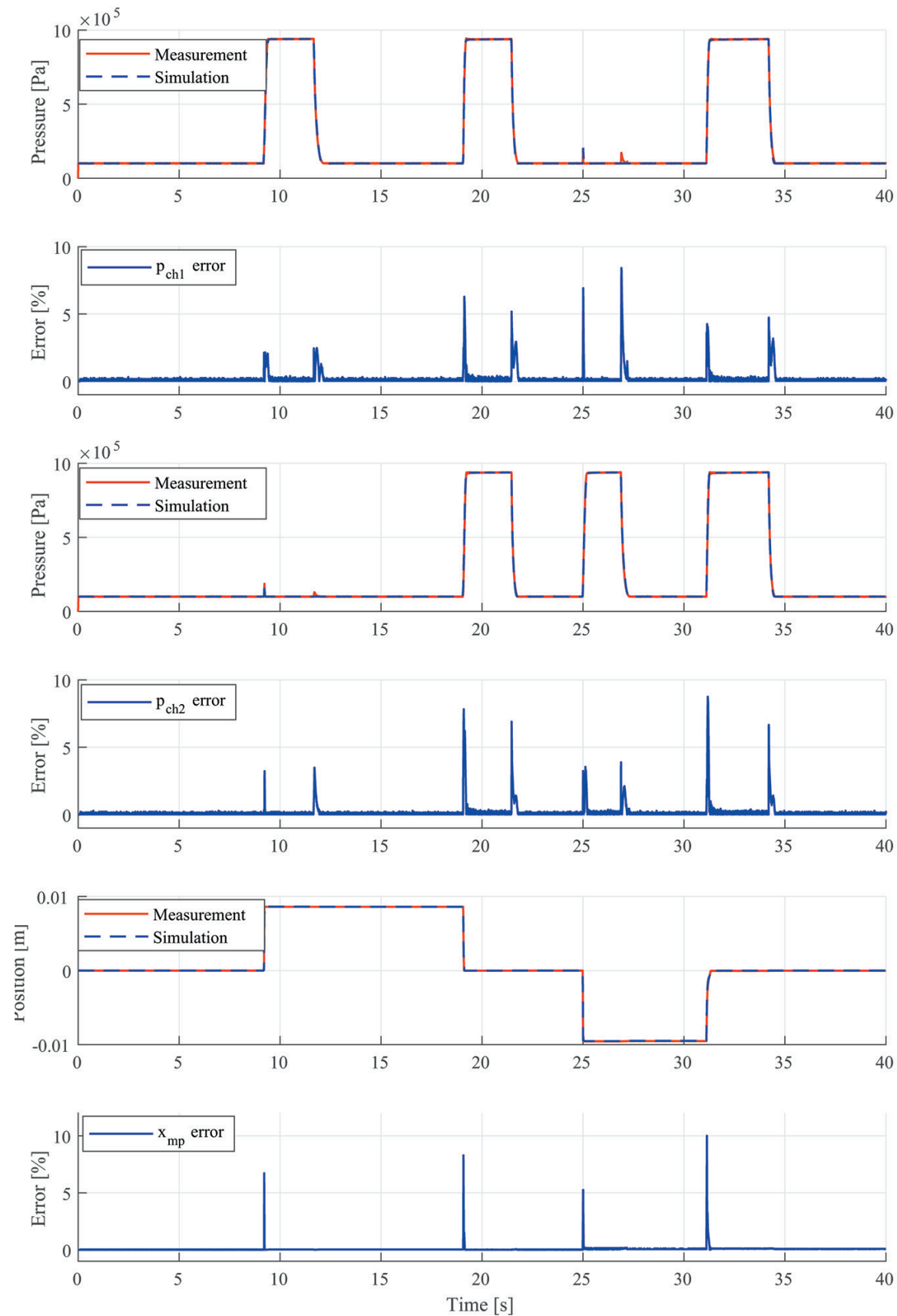

Fig. 10 Deviances of the validated outputs 
in both sides of the cylinder; however, it is possible that in the left side the skirt geometry limits the cross-section of the flow instead of the port.

In spite of the flaws of the model it can be said that it became able to simulate the system behavior with acceptable accuracy, as its unknown parameters were tuned to minimize its sum squared error compared to the measurement.

The model accuracy could be further improved by more detailed validation process (e.g. more measured outputs, and more validated sub models), or by modeling simplified physical phenomena in details, such as the friction or the heat transfer. Currently the heat transfer areas are assumed to be constant, but they could be calculated based on the piston positions. There could also be a more complex friction model implemented, such as the previously mentioned Stribeck model (which is a steady state model), or even dynamic models, for instance the LuGre model, or the seven-parameter friction model. While these

\section{References}

Bringmann, E., Krämer, A. (2008) "Model-Based Testing of Automotive Systems", In: 2008 1st International Conference on Software Testing, Verification, and Validation, Lillehammer, Norway, pp. 485-493. https://doi.org/10.1109/ICST.2008.45

Deng, W., Lee, Y. H., Zhao, A. (2008) "Hardware-in-the-loop simulation for autonomous driving", In: 2008 34th Annual Conference of IEEE Industrial Electronics, Orlando, USA, pp. 1742-1747. https://doi.org/10.1109/IECON.2008.4758217

Duncan, K. R., Etienne-Cummings, R. (2017) "A model based approach for realizing a safe wireless biotelemetry system", In: 2017 IEEE International Symposium on Circuits and Systems (ISCAS), Baltimore, USA, pp. 1-4. https://doi.org/10.1109/ISCAS.2017.8050366

Ishaku, J., Lotfi, N., Zomorodi, H., Landers, G. R. (2014) "Controloriented modeling for open-cathode fuel cell systems", In: 2014 American Control Conference, Portland, USA, pp. 268-273. https://doi.org/10.1109/ACC.2014.6859221

Kako, J., Soejima, S., Ohata, A. (2007) "Efficient Engine Development Using Model Based Development (MBD)" In: 2007 Chinese Control Conference, Hunan, China, pp. 603-607. https://oi.org/10.1109/CHICC.2006.4347173

Mihály, A., Baranyi, M., Németh, B., Gáspár, P. (2017) "Tuning of Look-ahead Cruise Control in HIL Vehicle Simulator", Periodica Polytechnica Transportation Engineering, 45(3), pp. 157-161. https://doi.org/10.3311/PPtr.9897

Németh, B., Gáspár, P. (2010) "Vehicle modeling for integrated control design", Periodica Polytechnica Transportation Engineering, 38(1), pp. 45-51. https://doi.org/10.3311/pp.tr.2010-1.08

Ogata, H., Katayama, T. (2010) "Skill Standards for Model Based Development Engineers in the Automotive Industry", IFAC Proceedings Volumes, 42(24), pp. 240-244. https://doi.org/10.3182/20091021-3-JP-2009.00044 modifications could increase the model accuracy, they would also increase the calculation cost and the number of unknown parameters.

Future work will focus on the complementing of the actuator model with a synchronizer model - which takes into consideration the engagement probability of the synchromesh mechanism - and on the development of Neural Network based controllers to achieve the position control of the system.

\section{Acknowledgement}

EFOP-3.6.3-VEKOP-16-2017-00001: Talent management in autonomous vehicle control technologies. The Project is supported by the Hungarian Government and co-financed by the European Social Fund

The project presented in this article is supported by the Magyar Automuszaki Felsooktatasert Alapitvany.

Ohata, A. (2009) "Control design in Model-Based Development and new SICE benchmark control design problem", In: 2009 ICCAS-SICE, Fukuoka, Japan, pp. 1870-1872. [online] Available at: https://ieeexplore.ieee.org/document/5332842 [Accessed: 23 April 2019]

Petrone, F., Sandroni, C., Farina, M., Scattolini, R., Guagliardi, A., Veneroni, A. (2012) "Object-oriented modeling of a power network for model-based voltage control", In: CIRED 2012 Workshop: Integration of Renewables into the Distribution Grid, Lisbon, Portugal, pp. 1-4. https://doi.org/10.1049/cp.2012.0833

Rabinovici, R., Tokar, D., Baimel, D. (2012) "Medium voltage multilevel inverters: Hardware-in-the-loop (HIL) simulations", In: 2012 IEEE 27th Convention of Electrical and Electronics Engineers in Israel, Eilat, Isreal, pp. 1-5. https://doi.org/10.1109/EEEI.2012.6377130

Santillan-Galvan, R., Martinez-Garcia, J. C., Garrido-Moctezuma, R. (2004) "Control-oriented modeling of an electrically actuated piston: an object-oriented approach", In: 2004 5th Asian Control Conference (IEEE Cat. No.04EX904), Melbourne, Australia, pp. 288-295. [online] Available at: https://ieeexplore.ieee.org/document/1425971 [Accessed: 23 April 2019]

Szabó, Á., Bécsi, T., Gáspár, P., Aradi, S. (2018) "Control oriented modeling of an electro-pneumatic gearbox actuator", In: 2018 European Control Conference (ECC), Limassol, Cyprus, pp. 2623-2628. https://doi.org/10.23919/ECC.2018.8550589

Wakitani, S., Yamamoto, T. (2017) "Practice of model-based development for automotive engineers", In: 2017 IEEE Frontiers in Education Conference (FIE), Indianapolis, USA, pp. 1-4. https://doi.org/10.1109/FIE.2017.8190678 